\title{
miR-26a suppresses osteosarcoma migration and invasion by directly targeting HMGA1
}

\author{
JIANYONG LIU ${ }^{1}$, BO MI $^{2}$, YI WANG ${ }^{3}, \mathrm{CHUNLING} \mathrm{SHI}^{4}$, XIUFANG MI $^{5}$, \\ YINGYING LU ${ }^{6}$ and PEILIN YU ${ }^{7}$
}

\begin{abstract}
${ }^{1}$ Department of Orthopedics, People's Hospital of Weifang, Weifang, Shandong 261000; ${ }^{2}$ Department of Medicine, The First People's Hospital of Jinan, Jinan, Shandong 250000; ${ }^{3}$ Department of Respiratory Medicine,

The People's Hospital of Zhangqiu Area, Jinan, Shandong 250200; ${ }^{4}$ Department of Neurology,

People's Hospital of Rizhao, Rizhao, Shandong 276800; ${ }^{5}$ Department of Medicine, The People's Hospital of Zhangqiu Area, Jinan, Shandong 250200; ${ }^{6}$ Department of Clinical Laboratory, People's Hospital of Rizhao, Rizhao, Shandong 276800;

${ }^{7}$ Department of Orthopedics, Qingdao Hiser Medical Center, Qingdao, Shandong 266033, P.R. China
\end{abstract}

Received September 15, 2017; Accepted February 28, 2018

DOI: $10.3892 / 01.2018 .8359$

\begin{abstract}
Osteosarcoma (OS) is identified as the most commonly diagnosed malignant cancer of bone, and has approximately three million new cases annually. miR-26a plays an important role in the development of various types of cancer. We investigated whether miR-26a can regulate the migration and invasion of OS by targeting high-mobility group A1 HMGA1. Western blot analysis was used to identify the changes of protein levels. Reverse transcription-quantitative PCR was used to test expression levels of genes and miR-26a. Luciferase reporter assay was used to test the specific target gene of miR-26a. Transwell assay was employed to determine the migration and invasion of OS cell lines. In the present study, miRNA-26a was frequently downregulated in OS tissues and cells. Overexpression of miR-26a inhibited cell migration and invasion in vitro. In addition, miR-26a downregulated HMGA1 by targeting its 3'-UTR and knockdown of HMGA1 significantly suppressed the migration and invasion of two osteosarcoma cell lines in vitro. miR-26a suppressed the migration and invasion of OS cells by targeting HMGA1, suggesting that miR-26a/HMGA1 axis provides a new prospective therapeutic strategy for OS.
\end{abstract}

\section{Introduction}

Osteosarcoma (OS) is a common malignant bone cancer type, with approximately 3,000,000 new cases annually (1). This type of cancer often occurs in adolescents and children, and

Correspondence to: Dr Peilin Yu, Department of Orthopedics, Qingdao Hiser Medical Center, 4 Renmin Street, Qingdao, Shandong 266033, P.R. China

E-mail: qec829@163.com

Key words: HMGA1, invasion, migration, miR-26a, osteosarcoma individuals over the age of 50 years are also susceptible to the disease (2). OS can be treated through surgery, chemotherapy and radiation and it is reported that the newly developed immunotherapies, such as chimeric antigen receptor-engineered $\mathrm{T}$ cells or immune checkpoint obstruction, can be employed to treat OS (3). Combination chemotherapies play an important role in the treatment of OS (4). Although almost all of the patients can accept the resection surgery, the OS recurrences remain high and often metastasize (5), and less than $20 \%$ of patients survive four years after recurrence $(6,7)$. Therefore, it is imperative to develop a novel therapeutic strategy to treat osteosarcoma more effectively.

MicroRNAs (miRNAs) are 21-22 nucleotide long non-coding RNAs and more than 1,000 miRNAs are expressed and function in human cells (8). miRNAs have been proven to play important roles in many biological processes, such as apoptosis, proliferation and differentiation (9). miRNAs regulate translation or splicing of their target mRNAs by binding to their 3'-UTRs (10). miR-26a is one of these miRNAs. Ma et al reported that the downregulation of miR-26a increased hepatocellular carcinoma growth and pulmonary metastasis (11). It was reported that miR-26a was significantly downregulated in bladder cancer (BC) tissues, and that the upregulation of miR-26a could suppress $\mathrm{BC}$ cell migration and invasion by modulating procollagenlysine, 2-oxoglutarate 5-dioxygenase (12). However, no relative evidence of miR-26a in osteosarcoma cells has been documented thus far.

The high-mobility group A (HMGA) family is comprised of three proteins: HMGA1a, HMGA1b and HMGA2 (13). HMGA proteins do not possess transcriptional ability, but they can change the chromatin structure to regulate the expression of various genes $(14,15)$. HMGA family proteins play pivotal roles in various biological processes, such as proliferation, differentiation, and chromatin structure (16). The expression levels of HMGA proteins are very low in normal tissues and cells. By contrast, a higher expression of HMGA is the hallmark of cancer $(17,18)$. Puca et al reported that HMGA1 was 
upregulated in colon tumor stem cell lines compared with the normal tissues (19). They identified that HMGA1 silencing could contribute to stem cell quiescence by increasing p53 levels (19). The overexpression of HMGA1 could increase cell proliferation and the stemness-related genes of the A2780 ovarian cancer stem line (20). Recent research has shown that HMGA1 acted as a target gene of miR-142 in regulating OS cell growth (21). However, whether HMGA1 was a target of miR-26a in regulating OS cell migration and invasion remains to be determined.

In the present study, we aimed to detect the role of miR-26a and HMGA1 in OS. We also explored the mechanism of miR-26a in regulating OS progression. The results showed that miRNA-26a was frequently downregulated in OS tissues and cells and the re-expression of miR-26a could suppress cell migration and invasion in vitro. Moreover, we identified that the expression of HMGA1 was decreased by miR-26a through targeting its 3'-UTR and knockdown of HMGA1 inhibited the migration and invasion of OS cells. Taken together, our study provides a new potential therapeutic target for OS treatment.

\section{Materials and methods}

Tissues and cell lines. Fifty-two pairs of human OS samples were obtained from patients who underwent surgery at the People's Hospital of Weifang (Weifang, China) from January, 2010 to December, 2016. All the patients provided written informed consent to participate in the study. Tissues obtained were immediately frozen in liquid nitrogen, stored at $-80^{\circ} \mathrm{C}$. This study was approved by the Ethics Committee of People's Hospital of Weifang.

Osteosarcoma cell line U2OS differentiated cell groups have high migratory capacity, and the normal human osteoblastic cell line hFOB 1.19 rarely divide, or divide at the limit of $39.5^{\circ} \mathrm{C}$ low migratory capacity. The cells were cultured in Dulbecco's modified Eagle's medium (Gibco; Thermo Fisher Scientific, Inc., Waltham, MA, USA) supplemented with $10 \%$ fetal bovine serum (Biowest, South America Origin), $100 \mathrm{U} / \mathrm{ml}$ penicillin and $100 \mathrm{mg} / \mathrm{ml}$ streptomycin (Sigma-Aldrich, St. Louis, MO, USA; Merck KGaA, Munich, Germany, respectively) in a humidified incubator with $5 \%$ $\mathrm{CO}_{2}$ at $37^{\circ} \mathrm{C}$.

Oligonucleotide transfection. The miR-26a mimic, inhibitor or negative control was transfected into cells for $48 \mathrm{~h}$ using Lipofectamine ${ }^{\circledR} 2000$ according to the manufacturer's protocols. The primer sequences used were: miR-26a mimic forward, UUCAAGUAAUCCAGGAUAGGCU, and reverse, CCUAUCCUGGAUUACUUGAAUU. miR-26a inhibitor forward, ATGTTCTTTAATGTCGGGAGC, and reverse, AA AGAATTCTGCCCGTGAC. To overexpress HMGA1, vectors containing HMGA1 were transfected into cells for $48 \mathrm{~h}$ using RNAiMAX (Invitrogen; Thermo Fisher Scientific, Inc., Waltham, MA, USA) according to the manufacturer's protocols.

Western blot analysis. The cells were harvested and lysed with RIPA lysis buffer (Beijing Solarbio Science \& Technology Co., Ltd., Beijing, China). Proteins were separated by $10 \%$
SDS-PAGE gel and transformed onto PVDF membranes (Invitrogen; Thermo Fisher Scientific, Inc.). The membranes were incubated with the primary antibody HMGA1, (dilution, 1:1,000; cat. no. ab168260), $\beta$-actin, (dilution, 1:1,000; cat. no. ab8227) (Abcam, Cambridge, UK) overnight at $4^{\circ} \mathrm{C}$ after being blocked with 5-10\% skimmed milk at temperature for $2 \mathrm{~h}$. The membrane was then incubated with secondary antibody (dilution, 1:2,000; cat. no. sc-516102-CM; Santa Cruz Biotechnology, Inc., Dallas, TX, USA) conjugated to horseradish peroxidase goat anti-mouse at room temperature for $2 \mathrm{~h}$. Signals were visualized using the enhanced chemiluminescence kit (EMD Millipore, Billerica, MA, USA). The intensities of individual bands were analyzed by densitometry using ImageJ software (National Institutes of Health, Bethesda, MD, USA) and normalized to the $\beta$-actin level.

Reverse transcriptase-quantitative PCR. Total RNA was extracted from cells or tissues using TRIzol reagent (Invitrogen; Thermo Fisher Scientific, Inc.) according to the manufacturer's instruction. Complementary DNA was synthesized using PrimeScript RT reagent kit (Takara, Dalian, China) and RT-qPCR was performed using SYBR Premix Ex Taq (Takara Biotechnology Co., Ltd., Dalian, China) with the Stratagene Mx3000P real-time PCR system (Agilent Technologies, Inc., Santa Clara, CA, USA). The primer sequences used were: miR-26a forward, TTGGATCCGTCAGAAATTCTCTCCC GAGG, and reverse, GGTCTAGATGTGAACTCTGGTGTT GGTGC. HMGA1 forward, GGCCCAAATCGACCATAAA GG, and reverse, GGACAAATCATGGCTACCCCT. U6 forward, GCTTCGGCAGCACATATACTAAAAT, and reverse, CGCTTCACGAATTTGCGTGTCAT. $\beta$-actin forward, CGTGACATTAAGGAGAAGCTG, and reverse, CT GAAGCATTTGCGGTGGAC. The thermocycler conditions were: $95^{\circ} \mathrm{C}$ for $5 \mathrm{~min}$, followed by 40 cycles of denaturation at $95^{\circ} \mathrm{C}$ for $15 \mathrm{sec}$ and an annealing/elongation step at $60^{\circ} \mathrm{C}$ for 30 sec. U6 and $\beta$-actin were used as internal controls to standardize miRNA and mRNA, respectively. The $2^{-\Delta \Delta \mathrm{Cq}}$ method was used to detect the relative expression of miR-26a and HMGA1.

Luciferase reporter assay. HMGA1-3'UTR was amplified and cloned into pmir-GLO vector (Promega Corporation, Madison, WI, USA). Mutation of HMGA1-3'UTR was generated using QuikChange Site-Directed Mutagenesis kit (Stratagene; Agilent Technologies, Inc.). For luciferase reporter assay, pmir-GLO-HMGA1-3'UTR-WT or mutant plasmid plus miR-26a mimic or miR-control were transfected into U2OS cells. After $48 \mathrm{~h}$ of transfection, cells were collected and relative luciferase activity was measured by the dual-luciferase reporter assay system (Promega Corporation). The firefly luciferase activity was normalized to Renilla luciferase activities.

Migration and invasion assay in vitro. Cell migration and invasion assays were performed in a 24-well Transwell plate (pore size, $8 \mu \mathrm{m}$; Corning Incorporated, Corning, NY, USA). Cells $\left(1 \times 10^{5}\right)$ were resuspended in $200 \mu 1$ of serum-free medium and plated onto the upper chamber. The lower chamber was filled with $15 \%$ fetal bovine serum as a 
A

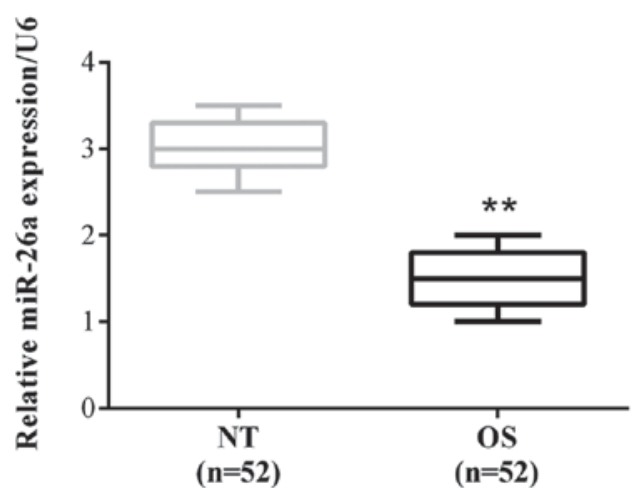

C

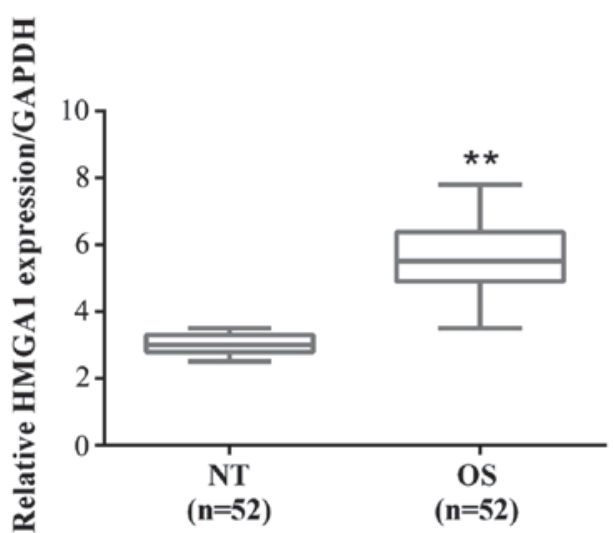

B

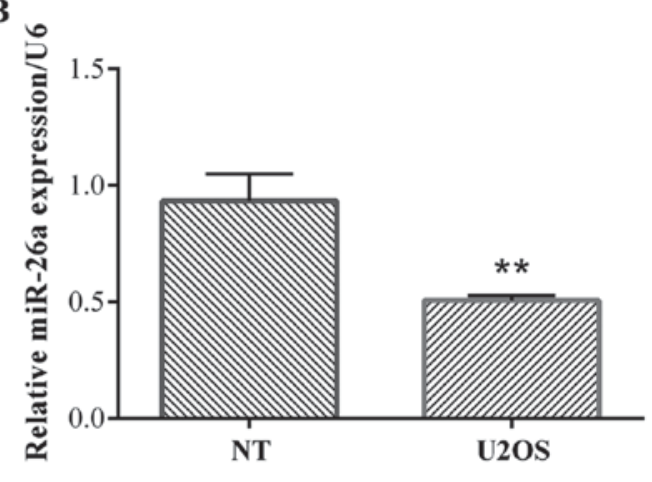

D

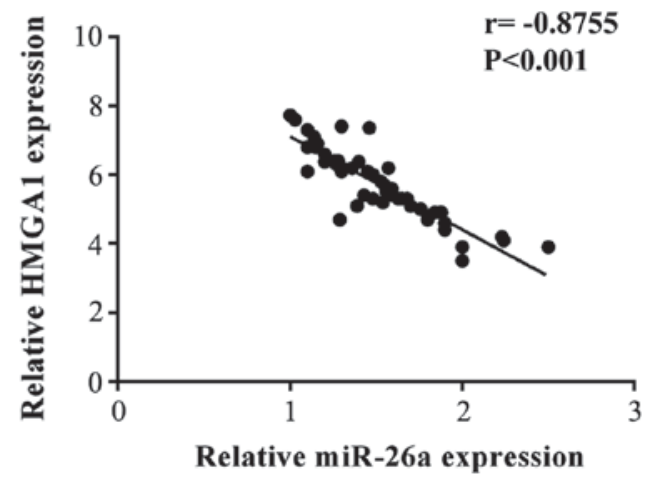

Figure 1. miR-26a is downregulated and HMGA1 is upregulated in OS tissues and cells. (A) miR-26a expression levels in OS tissues and their corresponding adjacent non-tumor tissues were determined using RT-qPCR ${ }^{(* *} \mathrm{P}<0.01$ vs. NT). (B) miR-26a expression levels in U2OS cells were also tested using RT-qPCR $\left({ }^{* *} \mathrm{P}<0.01\right.$ vs. NT). (C) HMGA1 expression levels in OS tissues and their corresponding adjacent non-tumor tissues were determined using the RT-qPCR $\left({ }^{* *} \mathrm{P}<0.01\right.$ vs. NT). (D) The relationship between miR-26a and HMGA1 expression was shown by regression analysis $(\mathrm{r}=-0.8755, \mathrm{P}<0.001)$. HMGA1, targeting high-mobility group A1; OS, osteosarcoma; RT-qPCR, reverse transcription-quantitative polymerase chain reaction; U2OS, two osteosarcoma cell line.

chemoattractant. For the invasion assay, the upper chamber was pre-coated with Matrigel (dilution, 1:3; BD Biosciences, San Jose, CA, USA). After incubation for $24 \mathrm{~h}$, the cells on the upper chamber were removed, and the migratory and invasive cells on the lower chamber were fixed using formalin and stained with $0.1 \%$ crystal violet $30 \mathrm{~min}$ at room temperature. Finally, the cells were photographed using a Leica DC 300F positive microscope (Leica Microsystems Imaging Solutions Ltd., Cambridge, UK) at x20 magnification.

Statistical analysis. The results are presented as the mean \pm standard deviation (SD). Statistical analysis was conducted using the SPSS 17.0 (SPSS, Inc., Chicago, IL, USA). Each experiment was repeated at least three times. Student's t-test or post hoc test after one-way analysis of variance (ANOVA) in SPSS were used to analyze the differences between the groups. Correlation between mRNA and miRNA were estimated using the Spearman's correlation method. $\mathrm{P}<0.05$ was considered to indicate a statistically significant difference.

\section{Results}

miRNA-26a is frequently downregulated and HMGAl is upregulated in OS. To determine the role of miR-26a in OS, we tested the expression level of miR-26a in OS and normal tissues in a group of $52 \mathrm{OS}$ patients by reverse transcription-quantitative polymerase chain reaction (RT-qPCR). The results showed that a relative miR-26a expression was frequently decreased in OS tissues compared with their adjacent non-tumor (NT) tissues (Fig. 1A). Then, we detected the expression of miR-26a in OS cells. The results showed that miR-26a was also significantly decreased compared with normal bone cells (hFOB 1.19) in both OS cell lines (Fig. 1B). We did not detect the expression of miR-26a in the various tumor stages.

HMGA1 expression in OS and normal tissues in a group of 52 OS patients by RT-qPCR. The results stated that the relative HMGA1 expression was frequently increased in OS tissues compared with their adjacent NT tissues (Fig. 1C). The relationship between miR-26a and HMGA1 expression was negatively correlated (Fig. 1D).

Overexpression of miR-26a suppresses cell migration and invasion in vitro. To investigate the potential role of $\mathrm{miR}-26 \mathrm{a}$ in the migration and invasion of OS, we selected U2OS cells for further investigation. Firstly, miR-26a mimic was transfected into U2OS cells to overexpress miR-26a. The successful overexpression of miR-26a was determined by RT-qPCR (Fig. 2A). Subsequently, we used a Transwell assay to detect the cell migration and invasion after U2OS cells were transfected with miR-26a mimic. Fig. 2B shows the overexpression of 
A

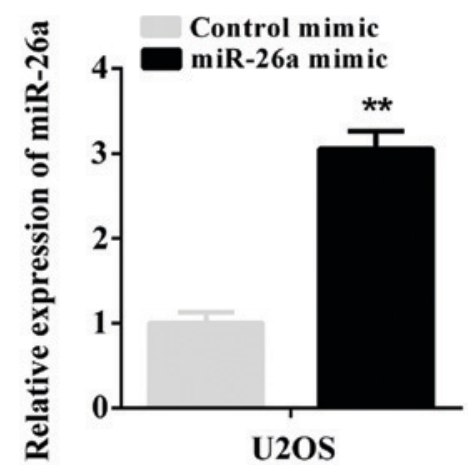

B

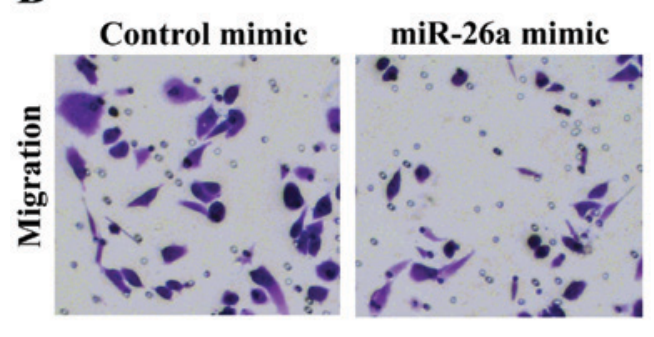

Control mimic

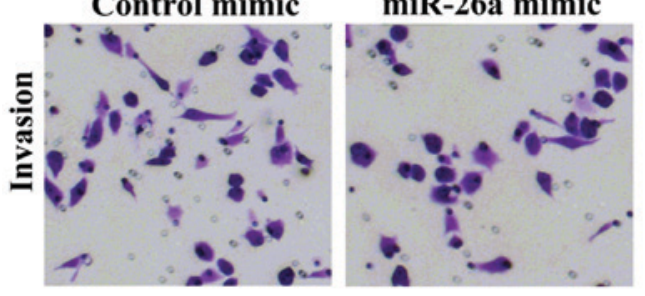

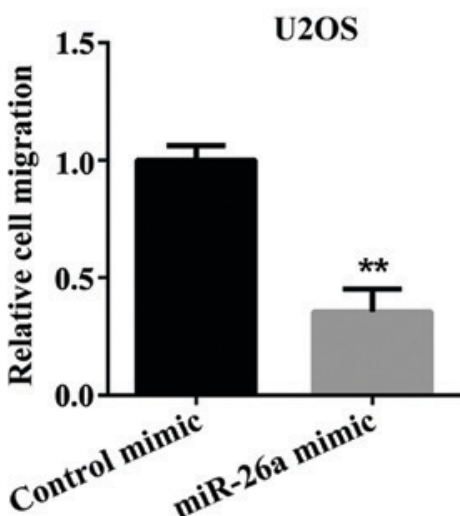

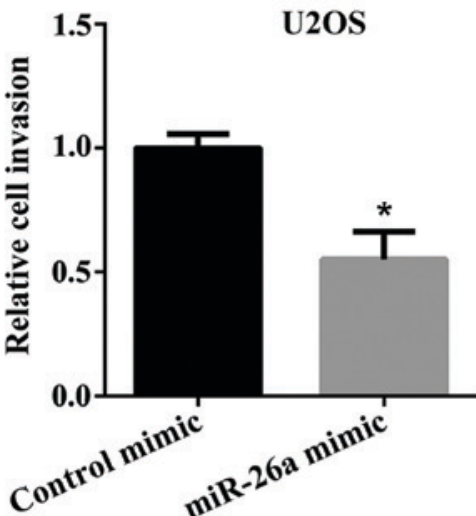

Figure 2. Re-expression of miR-26a inhibits cell migration and invasion. (A) U2OS cells were transfected with miR-26a mimic. The expression of miR-26a in U2OS cells was examined by RT-qPCR ( ${ }^{* *} \mathrm{P}<0.01$ vs. control mimic). (B) The migration and invasion of U2OS cells transfected with miR-26a mimic or negative control was tested by Transwell assay $\left({ }^{* *} \mathrm{P}<0.01,{ }^{*} \mathrm{P}<0.05\right.$ vs. control mimic). U2OS, two osteosarcoma cell line; RT-qPCR, reverse transcription-quantitative polymerase chain reaction.

miR-26a significantly suppressed cell migration and invasion. These findings indicated that miR-26a suppressed cell migration and invasion in vitro.

HMGA1 is identified as the target of miR-26a. To elucidate the potential mechanism of miR-26a in suppressing OS migration and invasion, we searched for the potential target genes of miR-26a using the bioinformatics algorithm TargetScan (http://www.targetscan.org/vert_71/). HMGA1, containing a potential binding site at 1317-1324 bp, was selected for experimental validation (Fig. 3A). Luciferase reporter assay showed that the luciferase activity was significantly reduced when U2OS cells were co-transfected with pmir-GLO-HMGA1-3'UTR (WT) and miR-26a $(\mathrm{P}<0.01)$. There was no effect on cells co-transfected with pmirGLO-HMGA1-3'UTR (MUT) and miR-26a (Fig. 3B). We further examined the protein expression of HMGA1 in U2OS cells after transfected with miR-26a mimic or miR-26a inhibitor by using western blot analysis. The results showed that overexpression of miR-26a reduced HMGA1 expression and knockdown of miR-26a increased HMGA1 expression (Fig. 3C). These findings indicated that HMGA1 was a direct target of miR-26a in OS cells.

Effect of HMGAI on OS cell migration and invasion regulated by miR-26a in vitro. Firstly, the HMGA1 siRNA or negative control was transfected into the U2OS cells to investigate the effect of HMGA1 on the OS cells. The expression of HMGA1 was detected by western blot analysis (Fig. 4A) and RT-qPCR (Fig. 4B). We then used a Transwell assay to test the relative cell migration and invasion in U2OS cells. The results showed that the migration and invasion of U2OS 
A

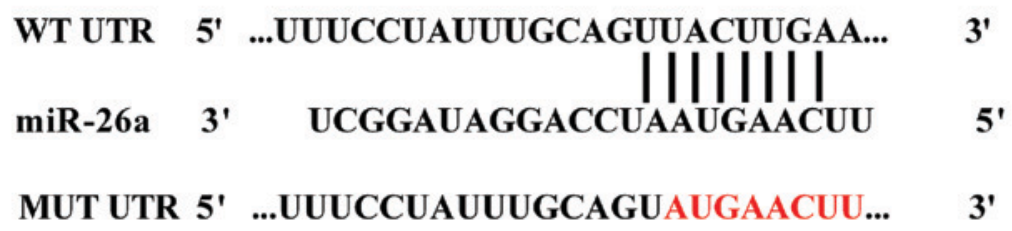

B
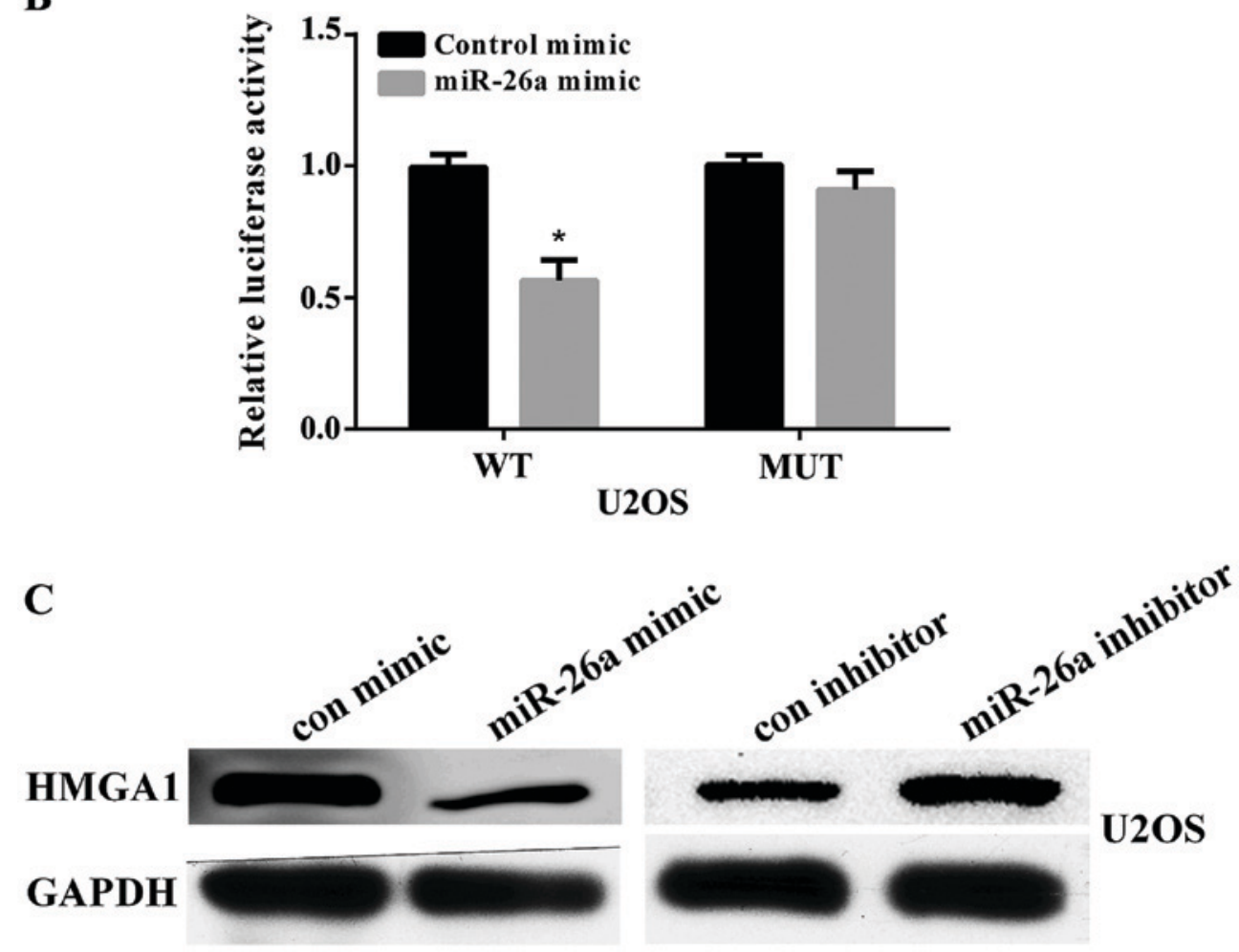

Figure 3. HMGA1 is the target of miR-26a and inhibited by miR-26a. (A) The binding site of HMGA1 for the miR26a was predicted by the TargetScan website. The mutated nucleotides are indicated in red. (B) The U2OS cells were co-transfected with the pmir-GLO-HMGA1-3'UTR (WT) or pmir-GLO-HMGA1-3'UTR (MT) luciferase reporter and miR-26a mimic. The luciferase activity was measured using the One-Glo luciferase assay instrument ("P $<0.05 \mathrm{vs}$. control mimic). (C) The U2OS cells was transfected with miR-26a mimic and inhibitor. The protein levels of HMGA1 in U2OS cells were determined using western blot analysis. HMGA1, targeting high-mobility group A1; U2OS, two osteosarcoma cell line; WT, wild-type; MUT, mutant-type.

cells transfected with the HMGA1 siRNA was significantly decreased compared with the cells transfected with the negative control (Fig. 4C and D). Then, we investigated the effect of HMGA1 on OS cell migration and invasion downregulated by miR-26a. We found that HMGA1 could reverse the inhibitory effect of miR-26a on OS cell migration and invasion (Fig. 4E and F).

\section{Discussion}

OS is the most common pattern of bone tumor (22). Although efforts have been made to explore the underlying mechanism of OS tumorigenesis, the survival rate of OS remains low (23). Therefore, it is imperative to identify new biomarkers for the development of effective therapeutic methods for OS.

In the present study, we explored the function of miR-26a and HMGA1 on human OS. Firstly, we examined the expression level of miR-26a in OS tissues and cells. The results showed that the expression level of miR-26a was significantly decreased in OS tissues and cells compared with the normal ones. Then we overexpressed miR-26a by transfecting the
miR-26a mimic into the U2OS cells. We found that overexpression of the miR-26a could suppress the invasion and migration of U2OS cells. Using the TargetScan online prediction and luciferase reporter assay, we further identified and confirmed that HMGA1 was the target of miR-26a, and miR-26a could suppress the expression of HMGA1.

The miR-26 family is comprised of miR-26a and miR-26b. They play important roles in biological processes, such as cell cycle and differentiation $(24,25)$. The miR-26a has been identified downregulated in various human cancers. Lu et al (26) identified that the miR-26a inhibits OS tumor growth in vivo and in vitro by regulating Jagged1, which is consistent with our study. The expression of miR-26a was decreased in esophageal squamous cell carcinoma (ESCC) tumor tissues compared with the adjacent normal tissues and the miR-26a could suppress the proliferation and metastasis of ESCC (27). In addition, miR-26a also plays a role in the metabolism of lipids and glucose, modulating insulin sensitivity in type 2 diabetes (28). The miR-26a also plays a crucial role in pancreatic cancer (29), cholangiocarcinoma (30), breast cancer (31) and non-small lung cancer (32). Yu et al (33) 
A

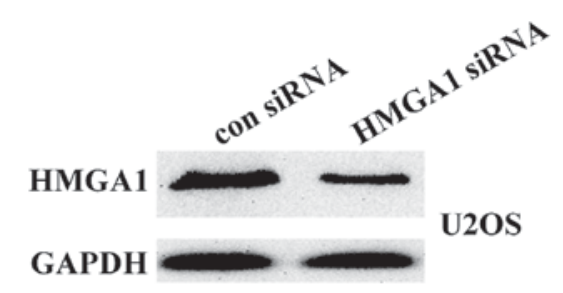

C

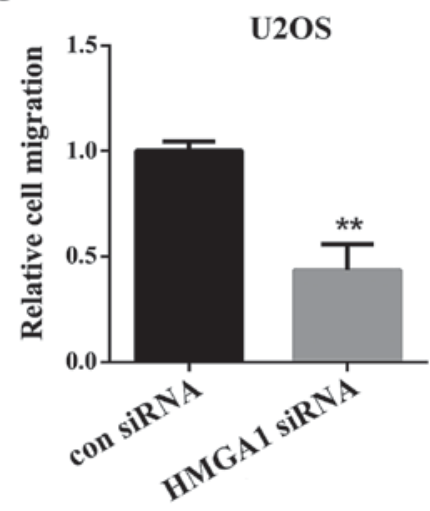

$\mathbf{E}$

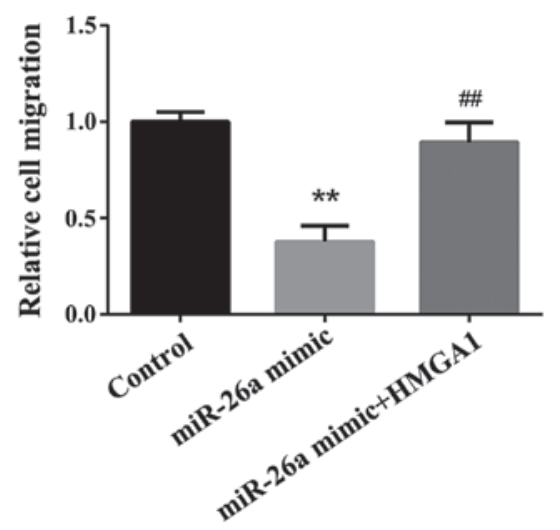

B

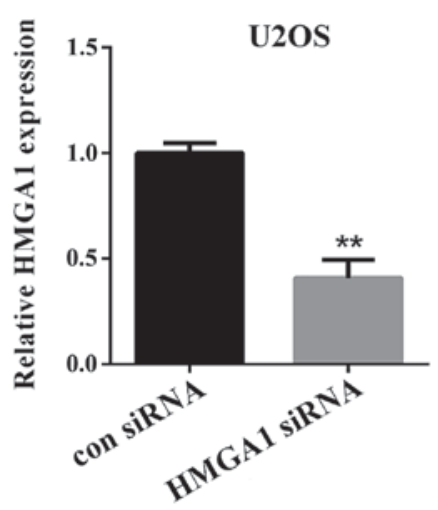

D

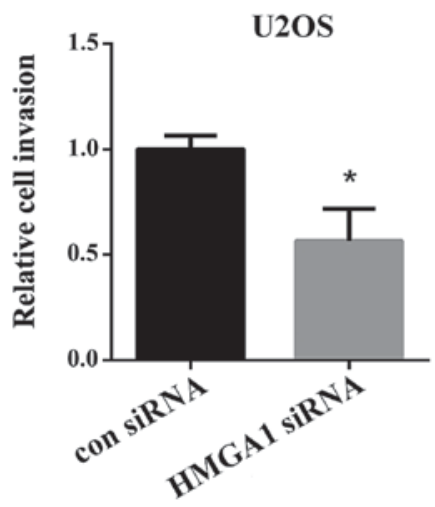

F

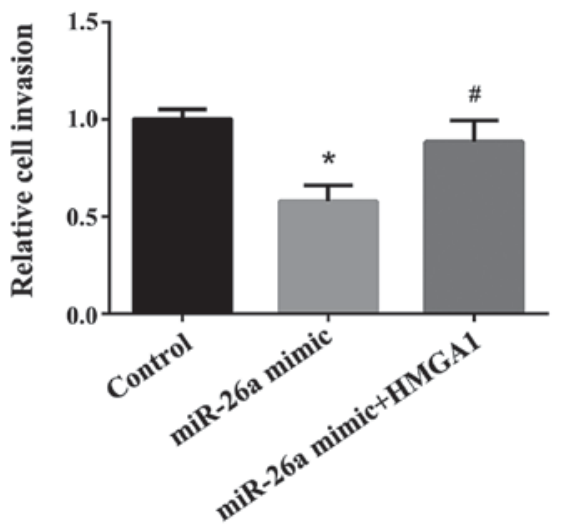

Figure 4. Knockdown of HMGA1 inhibits the migration and invasion of OS cells in vitro. (A and B) U2OS cells were transfected with HMGA1 siRNA or the negative control. The change of HMGA1 protein level and mRNA level was determined using the western blot analysis and RT-qPCR ( ${ }^{* *} \mathrm{P}<0.01 \mathrm{vs}$. con siRNA). $(\mathrm{C}$ and $\mathrm{D})$ The migration and invasion of the U2OS cells were tested using the MTT assay $\left({ }^{*} \mathrm{P}<0.05,{ }^{* * *} \mathrm{P}<0.01\right.$ vs. con siRNA). (E and F) U2OS cells were transfected with miR-26a mimic, both miR-26a mimic and HMGA1 or the negative control. The migration and invasion of the U2OS cells were tested using the MTT assay $\left({ }^{*} \mathrm{P}<0.05,{ }^{* *} \mathrm{P}<0.01\right.$ vs. con siRNA; ${ }^{\#} \mathrm{P}<0.05,{ }^{\# \#} \mathrm{P}<0.01$ vs. con siRNA). HMGA1, targeting high-mobility group A1; U2OS, two osteosarcoma cell line; RT-qPCR, reverse transcription-quantitative polymerase chain reaction; MTT, methyl thiazolyl tetrazolium assay.

reported that miR-26a was significantly upregulated in pituitary cancer tissues. There may exist other potential downstream targets of miR-26a in human OS.

HMGA1 can regulate various genes due to its ability to alter the chromatin structure. It is reported that the CCNE2 was a downstream target of HMGA1. The HMGA1 and CCNE2 regulate the cell migration through the Hippo core kinases in breast cancer (34). The HMGA1 activated cell stemness and crucial migration-related genes. The HMGA1 gene expression was related with the poor prognosis in breast cancer (35). HMGA1 overexpression is a hallmark of human cancer and exhibits a pivotal role in cell transformation (36). Our research is also consistent with that report. In the present study, we identified that the knockdown of HMGA1 significantly suppressed the migration and invasion of U2OS cells. In medulloblastoma (MB), cdc25A is a target of HMGA1 and HMGA1 interacts with cdc25A promoter. The knockdown of HMGA1 suppresses the MB cell invasion, migration and growth (37). The mutation of HMGA1 gene can influence its functions.

There are some drawbacks to the study. The number of patient samples is relatively small. Our research lacks clinical experiments. Thus, further study is required to address these aspects.

In conclusion, we identified that miRNA-26a was frequently downregulated in OS tissues and cells. The overexpression 
of miR-26a suppresses cell migration and invasion in vitro. Moreover, the HMGA1 is a target of miR-26a and the knockdown of HMGA1 inhibits the migration and invasion of U2OS cells. The miR-26a/HMGA1 axis provides new insight into the pathogenesis of OS and a biomarker for the treatment of OS.

\section{Acknowledgements}

Not applicable.

\section{Funding}

No funding was received.

\section{Availability of data and materials}

The datasets used and/or analyzed during the present study are available from the corresponding author on reasonable request.

\section{Authors' contributions}

JL and CS contributed to the conception of the study; BM and YW performed the data analyses; XM sorted out the experimental data; YL performed the data analyses and wrote the manuscript; PY helped perform the analysis with constructive discussions. All authors read and approved the final manuscript.

\section{Ethics approval and consent to participate}

This study was approved by the Ethics Committee of People's Hospital of Weifang (Weifang, China). All the patients provided written informed consent to participate in the study.

\section{Consent for publication}

Not applicable.

\section{Competing interests}

The authors declare that they have no competing interests.

\section{References}

1. Kansara M, Teng MW, Smyth MJ and Thomas DM: Translational biology of osteosarcoma. Nat Rev Cancer 14: 722-735, 2014.

2. Jo VY and Fletcher CD: WHO classification of soft tissue tumours: An update based on the 2013 (4th) edition. Pathology 46: 95-104, 2014

3. Isakoff MS, Bielack SS, Meltzer P and Gorlick R: Osteosarcoma: Current treatment and a collaborative pathway to success. J Clin Oncol 33: 3029-3035, 2015.

4. Ferrari S and Serra M: An update on chemotherapy for osteosarcoma. Expert Opin Pharmacother 16: 2727-2736, 2015.

5. Mirabello L, Troisi RJ and Savage SA: Osteosarcoma incidence and survival rates from 1973 to 2004: Data from the surveillance, epidemiology, and end results program. Cancer 115: 1531-1543, 2009.

6. Gorlick R, Janeway K, Lessnick S, Randall RL, Marina N and Committee COGBT; COG Bone Tumor Committee: Children's oncology group's 2013 blueprint for research: Bone tumors. Pediatr Blood Cancer 60: 1009-1015, 2013.

7. Geller DS and Gorlick R: Osteosarcoma: A review of diagnosis, management, and treatment strategies. Clin Adv Hematol Oncol 8: 705-718, 2010.

8. Krichevsky AM and Gabriely G: miR-21: A small multi-faceted RNA. J Cell Mol Med 13: 39-53, 2009.
9. Ambros V: The functions of animal microRNAs. Nature 431: 350-355, 2004.

10. Ha M and Kim VN: Regulation of microRNA biogenesis. Nat Rev Mol Cell Biol 15: 509-524, 2014.

11. Ma DN, Chai ZT, Zhu XD, Zhang N, Zhan DH, Ye BG, Wang CH, Qin CD, Zhao YM, Zhu WP, et al: MicroRNA-26a suppresses epithelial-mesenchymal transition in human hepatocellular carcinoma by repressing enhancer of zeste homolog 2 . J Hematol Oncol 9: 1, 2016.

12. Miyamoto K, Seki N, Matsushita R, Yonemori M, Yoshino H, Nakagawa Mand Enokida H: Tumour-suppressive miRNA-26a-5p and miR-26b-5p inhibit cell aggressiveness by regulating PLOD2 in bladder cancer. Br J Cancer 115: 354-363, 2016.

13. Johnson KR, Lehn DA and Reeves R: Alternative processing of mRNAs encoding mammalian chromosomalhigh-mobility-group proteins HMG-I and HMG-Y. Mol Cell Biol 9: 2114-2123, 1989.

14. Grosschedl R, Giese K and Pagel J: HMG domain proteins: Architectural elements in the assembly of nucleoprotein structures. Trends Genet 10: 94-100, 1994.

15. Thanos D and Maniatis T: The high mobility group protein HMG I (Y) is required for NF-kappa B-dependent virus induction of the human IFN-beta gene. Cell 71: 777-789, 1992.

16. Shah SN and Resar LM: High mobility group A1 and cancer: Potential biomarker and therapeutic target. Histol Histopathol 27: 567-579, 2012.

17. Chiappetta G, Avantaggiato V, Visconti R, Fedele M, Battista S, Trapasso F, Merciai BM, Fidanza V, Giancotti V, Santoro M, et al: High level expression of the HMGI (Y) gene during embryonic development. Oncogene 13: 2439-2446, 1996.

18. Pierantoni GM, Agosti V, Fedele M, Bond H, Caliendo I, Chiappetta G, Lo Coco F, Pane F, Turco MC, Morrone G, et al: High-mobility group A1 proteins are overexpressed in human leukaemias. Biochem J 372: 145-150, 2003.

19. Puca F, Colamaio M, Federico A, Gemei M, Tosti N, Bastos AU, Del Vecchio L, Pece S, Battista S and Fusco A: HMGA1 silencing restores normal stem cell characteristics in colon cancer stem cells by increasing p53 levels. Oncotarget 5: 3234-3245, 2014.

20. Kim DK, Seo EJ, Choi EJ, Lee SI, Kwon YW, Jang IH, Kim SC, Kim KH, Suh DS, Seong-Jang K, et al: Crucial role of HMGA1 in the self-renewal and drug resistance of ovarian cancer stem cells. Exp Mol Med 48: e255, 2016.

21. Xu G, Wang J, Jia Y, Shen F, Han W and Kang Y: MiR-142-3p functions as a potential tumor suppressor in human osteosarcoma by targeting HMGA1. Cell Physiol Biochem 33: 1329-1339, 2014.

22. Wan J,Zhang X, Liu T and Zhang X: Strategies and developments of immunotherapies in osteosarcoma. Oncol Lett 11: 511-520, 2016.

23. Velletri T, Xie N, Wang Y, Huang Y, Yang Q, Chen X, Chen Q, Shou P, Gan Y, Cao G, et al: P53 functional abnormality in mesenchymal stem cells promotes osteosarcoma development. Cell Death Dis 7: e2015, 2016.

24. Ji J, Shi J, Budhu A, Yu Z, Forgues M, Roessler S, Ambs S, Chen Y, Meltzer PS, Croce CM, et al: MicroRNA expression, survival, and response to interferon in liver cancer. N Engl J Med 361: 1437-1447, 2009.

25. Kota J, Chivukula RR, O'Donnell KA, Wentzel EA, Montgomery CL, Hwang HW, Chang TC, Vivekanandan P, Torbenson M, Clark KR, et al: Therapeutic microRNA delivery suppresses tumorigenesis in a murine liver cancer model. Cell 137: 1005-1017, 2009.

26. Lu J, Song G, Tang Q, Yin J,Zou C, Zhao Z, Xie X, Xu H, Huang G, Wang J, et al: MiR-26a inhibits stem cell-like phenotype and tumor growth of osteosarcoma by targeting Jagged1. Oncogene 36: 231-241, 2017.

27. Shao Y, Li P, Zhu ST, Yue JP, Ji XJ, Ma D, Wang L, Wang YJ, Zong Y, Wu YD, et al: MiR-26a and miR-144 inhibit proliferation and metastasis of esophageal squamous cell cancer by inhibiting cyclooxygenase-2. Oncotarget 7: 15173-15186, 2016.

28. Fu X, Dong B, Tian Y, Lefebvre P, Meng Z, Wang X, Pattou F, Han W, Wang X, Lou F, et al: MicroRNA-26a regulates insulin sensitivity and metabolism of glucose and lipids. J Clin Invest 125: 2497-2509, 2015.

29. Fu X, Jin L, Wang X, Luo A, Hu J, Zheng X, Tsark WM, Riggs AD, Ku HT and Huang W: MicroRNA-26a targets ten eleven translocation enzymes and is regulated during pancreatic cell differentiation. Proc Natl Acad Sci USA 110: 17892-17897, 2013.

30. Wang P and Lv L: miR-26a induced the suppression of tumor growth of cholangiocarcinoma via KRT19 approach. Oncotarget 7: 81367-81376, 2016 
31. Ma X, Dong W, Su Z, Zhao L, Miao Y, Li N, Zhou H and Jia L: Functional roles of sialylation in breast cancer progression through miR-26a/26b targeting ST8SIA4. Cell Death Dis 7: e2561, 2016.

32. Xu S, Wang T, Yang Z, Li Y, Li W, Wang T, Wang S, Jia L, Zhang $\mathrm{S}$ and Li S: miR-26a desensitizes non-small cell lung cancer cells to tyrosine kinase inhibitors by targeting PTPN13. Oncotarget 7: 45687-45701, 2016.

33. Yu C, Li J, Sun F, Cui J, Fang H and Sui G: Expression and clinical significance of miR-26a and pleomorphic adenoma gene 1 (PLAG1) in invasive pituitary adenoma. Med Sci Monit 22: 5101-5108, 2016.

34. Pegoraro S, Ros G, Ciani Y, Sgarra R, Piazza S and Manfioletti G: A novel HMGA1-CCNE2-YAP axis regulates breast cancer aggressiveness. Oncotarget 6: 19087-19101, 2015.

35. Pegoraro S, Ros G, Piazza S, Sommaggio R, Ciani Y, Rosato A, Sgarra R, Del Sal G and Manfioletti G: HMGA1 promotes metastatic processes in basal-like breast cancer regulating EMT and stemness. Oncotarget 4: 1293-1308, 2013.
36. Esposito F, De Martino M, Petti MG, Forzati F, Tornincasa M, Federico A, Arra C, Pierantoni GM and Fusco A: HMGA1 pseudogenes as candidate proto-oncogenic competitive endogenous RNAs. Oncotarget 5: 8341-8354, 2014.

37. Lau KM, Chan QK, Pang JC, Ma FM, Li KK, Yeung WW, Cheng AS, Feng H, Chung NY, Li HM, et al: Overexpression of HMGA1 deregulates tumor growth via cdc25A and alters migration/invasion through a cdc25A-independent pathway in medulloblastoma. Acta Neuropathol 123: 553-571, 2012.

This work is licensed under a Creative Commons Attribution-NonCommercial-NoDerivatives 4.0 International (CC BY-NC-ND 4.0) License. 